\title{
DIFFUSION: THEORETICAL COMPUTATIONS FOR NON-MAGNETIC STARS
}

\section{G. ALECIAN}

DAEC, URA173-CNRS, Observatoire de Paris, Section de Meudon, F-92195 MEUDON CEDEX, FRANCE

\begin{abstract}
We present the main aspects of diffusion calculations in the simplest realistic diffusion model for main-sequence $\mathrm{CP}$ stars. The results obtained with this kind of computations are generally used in modelling AmFm and $\mathrm{HgMn}$ stars. Important improvements in this field, essentially due to the new atomic data banks, may be expected in the near future.
\end{abstract}

\section{INTRODUCTION}

Diffusion is a rather weak process compared to the ones usually considered in a star: it needs long time scales, it is easily perturbed by any motions of the stellar fluid, it is sensitive to the presence of a magnetic field, and so on. However its effects are more than detectable since in some cases, even the stellar structure can be affected.

In a laboratory experiment about diffusion one would try to get ride of from any perturbation: the ideal situation being to have a pure hydrogen plasma with one trace element, without any mixing motion nor magnetic field, with known radiation field. Fortunately some stars like AmFm and $\mathrm{HgMn}$ stars are supposed to be more quiet than other stars (undetectable magnetic field and/or low rotation) and then this allows to test the diffusion model in realistic astrophysical situations.

The purpose of this talk is not to make a complete review of the works which have been done in that field but to show the different problems related, at the present time, to the diffusion calculations.

We shall present here some aspects of the computations which correspond to the minimum realistic model: diffusion in a main-sequence star (outside the nuclear reactions zone) with no or negligible magnetic field, with no or negligible rotation. The star is assumed stationary except with respect to the abundance stratification of trace elements.

Although this diffusion model is rather simple, there are still difficulties to overcome before to reach a reliable modelling of individual stars even for the less complex of them. 


\section{BASIC PROCESSES}

Building of abundance stratification is the result of many competing processes: absorption of photons which pushes the ions upward, gravity which attracts them downwards, "pure" diffusion which tries to smooth out any abundance inhomogeneity, thermal diffusion driven by the temperature gradient, turbulence which mixes the plasma and erases the patient work of diffusion, stellar wind which carries up the whole stellar plasma. In this section we shall briefly review the main processes entering the basic diffusion model computations.

\subsection{Diffusion of particles}

The basic formula giving the diffusion velocity $v_{i}$ of an ion $\mathrm{A}^{+\mathrm{i}}$ in a stellar plasma, has been often discussed by several authors, a detailed analysis of the present situation may be found in a recent review by Michaud (1992).

In its simplest form this velocity may be expressed as follows:

$$
v_{i}=D_{i}\left[-\left(1+\frac{D_{\text {turb }}}{D_{i}}\right) \frac{\nabla c_{i}}{c_{i}}+\alpha_{1} g_{i}^{\text {rad }}\left(c_{i}\right)-\alpha_{2} g+\alpha_{3} \frac{\nabla T}{T}\right]
$$

where $c_{i}$ is the concentration of the ion $\mathrm{A}^{+\mathrm{i}}, g^{\text {rad }}$ is the radiative acceleration, $g$ the gravity, $T$ the local temperature $D_{i}$ the microscopic diffusion coefficient, $D_{t u r b}$ the turbulent diffusion coefficient (see later on). All the terms in this expression (including the coefficients $\alpha$ ) depend implicitly on the depth in the star, $g_{i}^{\text {rad }}$ depends in addition, explicitly, on the ion concentration.

Actually, what is needed is the total diffusion velocity $v_{D}$ of an element since elements are, at a given depth in the star, in more than one state of ionization. This may be obtained simply by the way of weighted means over the individual ions velocities. However a precise computation needs a more sophisticated expression. For instance, in stellar atmospheres, detailed study must consider redistribution effects (Montmerle and Michaud 1976). More over, expression (1) supposes that the medium is a mixture of protons, electrons and a trace ion $\mathrm{A}^{+i}$, which is not in some cases, a good enough approximation for instance in the case of helium diffusion or when there is a strong gradient of the ratio HI/HII (ambipolar diffusion described by Babel and Michaud 1991).

\subsection{Mass loss}

In diffusion models, the effect of a mass loss has been considered by Vauclair (1975) to explain helium anomalies in He-rich stars. Indeed, helium overabundances in these stars cannot be explained by diffusion alone since this element is not supported by the radiation field and then must sink inside the star. To consider the combined effect of the global mass outflow and the downward diffusion of He gives a satisfactory explanation for the observed general tendency of He to be overabundant. Michaud et al (1983), Michaud and Charland (1987) have introduced the effects of mass-loss in the diffusion model for $\mathrm{A}$ and $\mathrm{F}$ stars this allows to give a coherent and better quantitative description of AmFm stars from helium diffusion (at the beginning of the process) until the 
appearance of metals anomalies. It is clear now that mass loss must be considered, more or less, in diffusion models for all CP stars.

Among the different parameters which regulate the elemental stratification, mass loss has a special status since it is defined by one unique quantity (the rate of mass loss) appearing very simply and explicitly in the equations (see later: $V_{w}$ in eq. (2)), and which applies for all the elements in the whole star (if no magnetic field). Its effects may be very strong as discussed later on in $\$ 4$.

\subsection{Effects of turbulent motions}

Turbulence affects the elements stratification through the term containing the coefficient $D_{\text {turb }}$ in (1) (Schatzman, 1969). There are two regimes: (i) strong turbulence which mixes completely the medium $\left(D_{t u r b} / D_{i} \gg 10^{4}\right.$ in (1), (ii) weak turbulence where diffusion and mixing may compete $\left(D_{t u r b} / D_{i} \leq 10^{4}\right)$. The first case (i) corresponds to what happens in a convection zone where diffusion cannot produce any abundance inhomogeneity, then $\nabla c$ remains equal to zero. The second case (ii) corresponds to a transition zone between turbulent and stable layers (bottom of convection zones), or weak turbulence induced by shear flows (related to stellar rotation) etc.

In the framework of the basic diffusion model considered here, we are concerned by the following questions: where are precisely the convection zones boundaries, how are convection zones linked to stable ones? The classical modelling of stellar envelopes cannot simply answer these questions. In CP stars helium is supposed to settle down and this must lead to the weakening (AmFm stars) or disappearance (HgMn stars) of the superficial convection zones. On the other hand, mixing may occur in slightly more extended region than the one predicted by the classical mixing-length theory (this is true also for normal stars). For instance, while overshooting is supposed to penetrate stable zones on about one pressure scale height, another process as internal waves may lead to more extended depth of mixing (Schatzman, 1991) but this last theory is not able for the moment to fix even a reliable order of magnitude for $D_{t u r b}$.

\subsection{Abundance stratifications}

The final product of the diffusion processes is the abundance stratification of elements which is obtained through the continuity equation:

$$
\partial_{t} c+\frac{1}{N_{H}} \nabla c N_{H}\left(v_{D}+V_{w}\right)=0
$$

where $\mathrm{c}$ is the element concentration, $N_{H}$ the hydrogen (neutral+protons) number density, $v_{D}$ the average diffusion velocity of the element, $V_{w}$ the velocity due to the stellar mass loss. Note that since $v_{D}$ has a non-trivial dependency on $c$ (see eq.(1)), eq. (2) must be integrated numerically. Note also that whether $V_{w}$ is always positive, $v_{D}$ may be negative and then cancellation of the total element velocity may occur at some places.

The numerical integration of eq. (2) is rather difficult to carry out because on the one hand, very short time scales are involved at the upper spatial boundary and on the other hand, calculation of $v_{D}$ is very CPU-time consuming. However there are at least two approximations that allow a first evaluation of what stratification may be expected. 
The simplest approximation is to determine (with respect to the depth) the concentration $c^{*}$ such as the radiative acceleration balances gravity: $g^{r a d}\left(c^{*}\right)=g$. This concentration $c^{*}$ is easy to be determined, but this approximation is rather crude since for instance it cannot account for the presence of the global mass outflow. However, there are at least two cases where this approximation has been successful: For manganese, Alecian and Michaud (1981) have predicted the maximum overabundance that can be supported by the radiation field in HgMn stars; the trend they have found is now confirmed by the new observations by Smith and Dworetsky (1990) and Smith (1992). There is also a good agreement for gallium comparing the maximum overabundance performed by Alecian and Artru (1987) to the observations by Smith (1992).

Another approximation consists in assuming that a stationary solution may be reached: $\nabla c N_{H}\left(v_{D}+V_{w}\right)=0$ every where in the star. Here the problem is that one cannot say whether this solution may be reached and if so, how long does it take to reach it: is this time compatible with time spent by the star on the main sequence? The occurrences of transient stages are also lost in a such approach but this is not so problematic when diffusion occurs in photosphere (where times scales are much smaller than below the superficial convection zone of AmFm stars).

\section{RADIATIVE ACCELERATIONS}

The radiative acceleration $g^{\text {rad }}$ is generally the dominant term in expression (1). A detailed computation of $g_{i}^{\text {rad }}$ consists in determining the total amount of momentum get from the radiation flux through photon absorptions by ions $\mathrm{A}^{+i}$. This needs to compute the radiation transfer for all the bound-bound and bound-free transitions of ion $\mathrm{A}^{+\mathrm{i}}$, at each layer and each time-step.

The main difficulties to overcome are: to compile the atomic data and to avoid huge computations.

\subsection{Atomic data}

We shall not insist here on the importance to have precise $(g f)$ and complete atomic data $\left(g f, \lambda, E_{i}\right.$ ) for the $g_{i}^{r a d}$ computations. We shall however emphasise on the significant improvements on this field that data, provided by works such as the Opacity project (Seaton, 1992), are going to bring (see Alecian et al 1992).

To have a complete data set raises another problem: how to deal with huge number of transitions. For instance OP data bank contains more than 77000 bound-bound transitions for FeXI ! Alecian and Michaud (1981) and Alecian et al (1992) have used a method for data selection and compression that may reduce in some cases by a factor up to 50 the number of transitions to be considered without impairing the final precision of the numerical results.

\subsection{Computations of the radiative accelerations}

In optically thin medium such as stellar atmospheres, the radiative accelerations must be computed in detail. A code computing synthetic spectra for instance may be used for that. The only approximation that may be done is the one consisting in selecting and compressing the atomic data (as mentioned in the previous section). In some cases LTE 
computations are sufficient (Alecian and Artru 1987). The fact that radiation transfer must be calculated in detail make this kind of computation very heavy and thus prevent, for the moment, any precise time-dependent computation of abundance stratification in the CP stars atmosphere.

In optically thick medium, absorption of photons may be treated locally and then the $g_{i}^{\text {rad }}$ determination is much easier. If one assumes that the lines profile are mainly lorentzian, the following analytical approximation (Alecian and Artru, 1990; Alecian et al, 1992) of $g_{i}^{r a d}$ is valid for bound-bound transitions:

$$
g_{i}^{r a d} \approx \beta(r) \Phi_{i}\left(1+\frac{c_{i}}{\delta(r) \Psi_{i}^{2}}\right)^{-\frac{1}{2}}
$$

where $\Phi_{i}$ and $\Psi_{i}$ are two parameters which gather the atomic properties of the ion $A^{+i}, \beta$ and $\delta$ are function of the local plasma conditions. In approximation (3) the dependency of $g_{i}^{r a d}$ on the stellar model, those on the atomic properties of $\mathrm{A}^{+1}$, and the element concentration are separated. In many cases one couple of $\Phi_{i}$ and $\Psi_{i}$ may be sufficient for each ion, to reach a rather good accuracy. Thus when concentration varies in eq.(2) there is no need to compute again the contribution of individual lines. This is also true changing the stellar model where only $\beta$ and $\delta$ have to be computed again.

This approximation has been checked by Alecian et al (1992) in the case of highly ionized iron for a main-sequence star with $\mathrm{T}_{\text {eff }}=8000 \mathrm{~K}$ (the blends are neglected). Figure 1 shows that expression (3) is very precise provided that $\Phi_{i}$ and $\Psi_{i}$ have been properly calibrated.

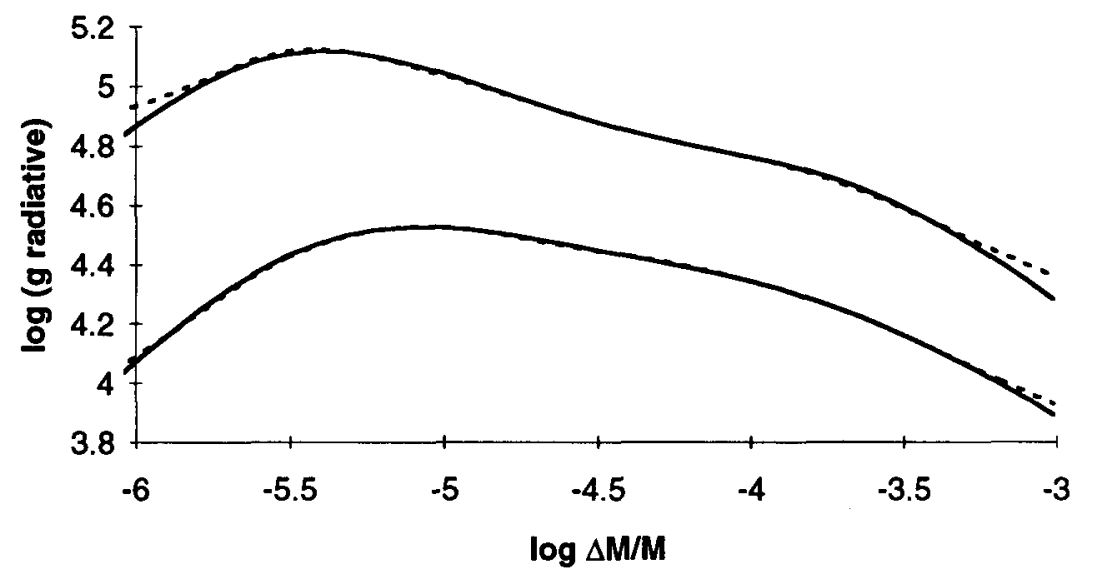

Figure 1. The detailed (solid lines) radiative accelerations of iron compared to those (dashed lines) computed with approximation (3). The lower curves are for solar abundance and the upper ones for $10^{-2}$ underabundance. 


\section{TIME-DEPENDENT DIFFUSION BELOW A SUPERFICIAL CONVECTION} ZONE

In this section we are concerned by how the superficial concentrations of stars may change during time. As already mentioned, this kind of computation cannot be done reliably for CP stars atmosphere even if some attempts have been made in that field. Therefore, we restrict ourselves to the case of diffusion below the superficial convection zone of AmFm stars where radiation transfer is easier to approximate.

Since convection zone ensures a complete mixing of elements, abundance anomalies created by diffusion just below this zone are brought up in the photosphere. To understand the chemical peculiarities of AmFm stars needs then to study the stratification processes below the convection zone. This kind of calculations (using eq. (2) with $V_{w} \neq 0$ ) have shown that abundances at the surface of $\mathrm{AmFm}$ stars have a non-monotonic time dependence (Michaud and Charland 1987; Alecian, 1990): element abundances vary along the life-time of these stars on the main sequence.

An example of such a computation (Alecian, 1992) is shown in Fig.(2) for Ca in an Am star with $\mathrm{T}_{\text {eff }}=7400 \mathrm{~K}, \log \mathrm{g}=4.05$ and a mass-loss rate of $10^{-14} \mathrm{M}_{\odot}$ per year. This preliminary result (the lack of atomic data has lead us to use the extrapolation method of Alecian and Artru, 1990) has been obtained with the approximation (3) which makes the computation of the radiative accelerations quick and precise. One may see that one second short phase of overabundance is followed by a third phase of underabundance after less than one billion years of diffusion.

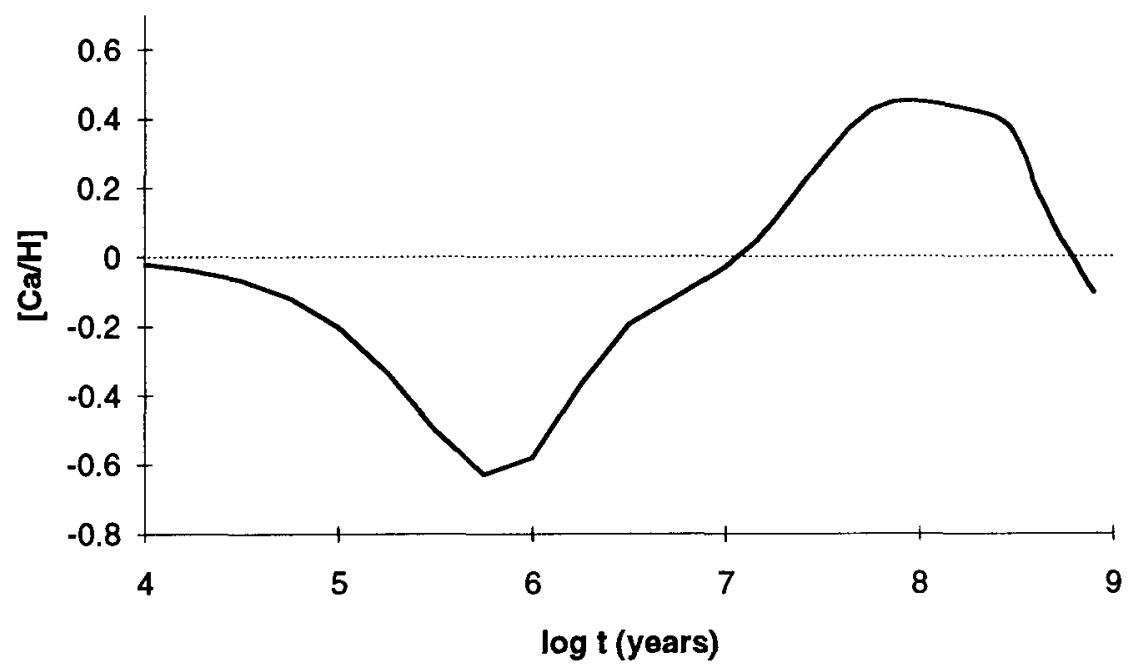

Figure 2. Calcium abundance during $10^{9}$ years of diffusion (preliminary result) 
Another aspect of the time-dependent diffusion is the high sensitivity of the anomalies according to the rate of mass-loss. This is illustrated in Fig. 3 which shows the evolution of calcium for three different rates $\left(0.9,1.0\right.$, and $3.010^{-14} \mathrm{M}_{\odot}$ per year).

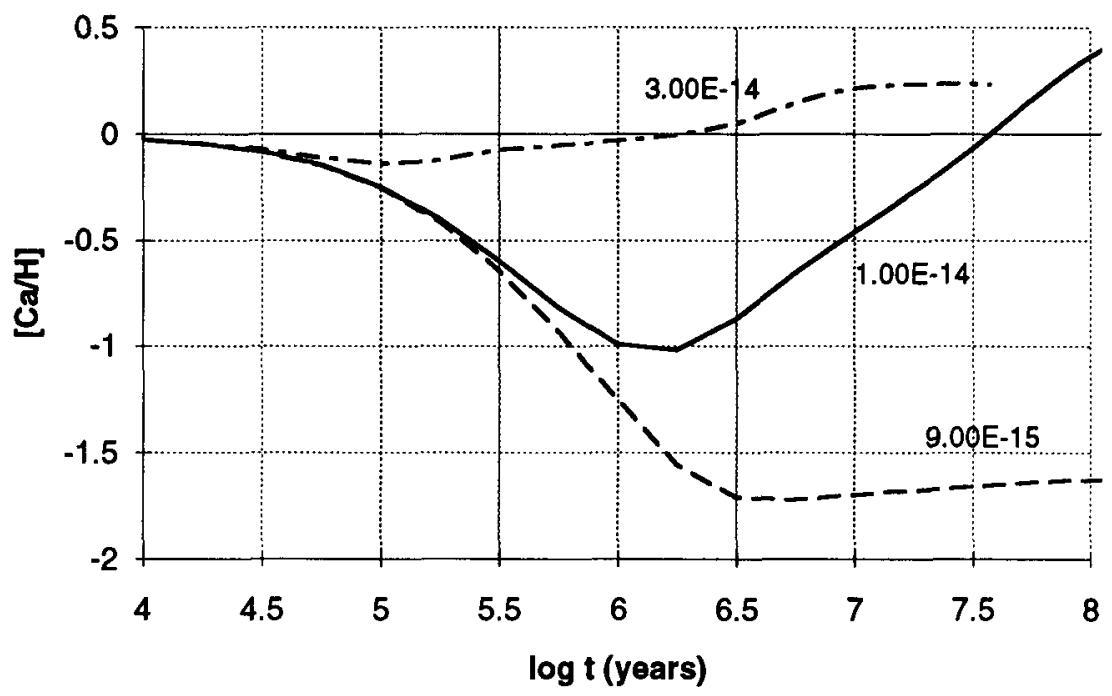

Figure 3. Calcium concentration in the convection zone (same stellar model as for Fig. 2) for three values of the mass-loss rate.

Such a sensitivity suggests that time-dependent calculations of diffusion should become a way for determining the mass loss rate of A-type stars which cannot be found by observational means at the present time.

\section{SOME CONCLUDING REMARKS}

A number of improvements have been made in theoretical computations of diffusion during the last decade: better approximations for the diffusion velocity (see Paquette $e t$ $a l, 1986$, for the diffusion coefficients) and for the radiative accelerations, more powerful numerical codes. However, the most impressive improvement is still coming: those due to the new atomic data banks. These new data allow to deal with better stellar models but over all (for diffusion computations) they will allow a precise computation of the radiative accelerations. We can estimate that the critical threshold of $30 \%$ of accuracy for radiative acceleration will be reachable. This is, according to Michaud and Charland (1987) the accuracy needed to make detail modelling of individual star. 
Such an accuracy will allow to better constrain the diffusion models even in the frame work of the simplest ones. It will be possible to test the different theories describing which kind of mixing may occur just below the convection zones (overshooting, internal waves...), what is the rate of mass-loss in A-type stars. And perhaps it will reveal some missing phenomenon in present models.

\section{REFERENCES}

Alecian, G.: 1990, IAU Symposium $\mathrm{N}^{\circ} 145$ On "Evolution Of Stars: The Photospheric Abundance Connection", Bulgaria, G. Michaud and A. Tutukov, p.11

Alecian, G.: 1992, IAU Colloquium 137 "Inside The Stars", Vienna, A. Baglin and W.W.Weiss

Alecian, G., Artru, M.-C.: 1987, Astron. Astrophys., 186, 223

Alecian, G., Artru, M.C.: 1990, Astron. Astrophys., 234, 323

Alecian, G., Michaud, G.: 1981, Astrophys.J., 245, 226

Alecian, G., Michaud, G., Tully, J.: 1992, Astrophys. J. (Submitted)

Babel J., Michaud, G.: 1991, Astron. Astrophys. (Accepted)

Michaud G.: 1992, IAU Colloquium 137 "Inside The Stars", Vienna, A. Baglin and W.W.Weiss

Michaud, G., Charland, Y.: 1987, Astrophys. J., 311, 326

Michaud, G., Tarasick, D., Charland, Y., Pelletier, C: 1983, Astrophys. J., 269, 239

Montmerle, T., Michaud, G.: 1976, Astrophys. J. Suppl., 31, 489

Paquette, C., Pelletier, C., Fontaine, G., Michaud, G.: 1986, Astrophys. J. Suppl., 61, 177

Schatzman, E.: 1969, Astron. Astrophys., 3, 331

Schatzman, E.: 1991, Mem. S.A.It., 62, 111

Seaton, M.J.: 1992, Iau Colloquium 137 "Inside The Stars", Vienna, A. Baglin and W.W.Weiss

Smith K.,C.: 1992, PhD (In press).

Smith K.,C., Dworetsky, M.M.: 1991, IAU Symposium N 145 , "Evolution Of Stars: The Photospheric Abundance Connetion", Bulgaria, G. Michaud and A. Tutukov, p.81

Vauclair, S.: 1975, Astron. Astrophys., 45, 233 